\title{
A Well-posed Model for Mixed-Sediment River Morphodynamics
}

\author{
Víctor Chavarrías ${ }^{1, *}$, Guglielmo Stecca ${ }^{2,3}$, Robert Jan Labeur ${ }^{1}$, and Astrid Blom ${ }^{1}$ \\ ${ }^{1}$ Faculty of Civil Engineering and Geosciences, Delft University of Technology, Delft, The Netherlands. \\ ${ }^{2}$ National Institute of Water and Atmospheric Research, Christchurch, New Zealand. \\ ${ }^{3}$ University of Trento, Trento, Italy.
}

\begin{abstract}
The mixed-size character of sediment is a necessary property to explain physical phenomena such as downstream fining or the presence of armor layers. The active layer model was developed to model mixed-size sediment in river morphodynamics. This model assumes that the topmost part of the bed, the active layer, has no vertical stratification and interacts with the flow. The substrate, below the active layer, only interacts with the active layer in case of aggradation or degradation. The active layer model has been used in morphodynamic modelling for more than four decades but under certain conditions it may become mathematically ill-posed. When a model becomes ill-posed, the solution presents unphysical oscillations and its predictive capabilities are lost. We present two alternatives to the active layer model. The first one retains the basic concepts and guarantees well-posedness by means of an additional parameter controlling the celerity of mixed-size sediment processes. The second solution yields a well-posed model by means of considering the sediment transport rate as a stochastic process rather than to adapt instantaneously to the flow. Both models provide reasonable results when compared to measured data from a laboratory experiment conducted under conditions in which the active layer model is ill-posed.
\end{abstract}

\section{Introduction}

To predict changes in bed elevation due to currents, commonly a (set of) equation(s) describing the flow (i.e., the Navier-Stokes equations or a simplification of them) is solved in combination with a (set of) equation(s) that accounts for the mass conservation of bed sediment. The Exner [1] equation represents the mass balance of the total amount of bed sediment. Hirano [2] developed the active layer model to account, not only for the changes in bed elevation, but also for the changes in grain size distribution. With this model it is possible to predict mixed-size sediment processes such as downstream fining in rivers [3] and bed surface armoring [4].

The combination of the flow and morphodynamic equations requires a closure model for sediment fluxes. This is often done using an algebraic relation for the sediment transport rate as a function of the bed shear stress [e.g. 5]. The use of such closure relation, however, implies that the sediment transport rate adapts instantaneously to changes in flow. In that

\footnotetext{
*e-mail: v.chavarriasborras@tudelft.nl
} 
Table 1. Different morphodynamic models in terms of: (1) the equation to update the bed, (2) the form of the equation, and (3) the type of sediment transport formulation.

\begin{tabular}{rrrr}
\hline Model & Bed Update Eq. & Eq. Form & Sed. Trans. Formulation \\
\hline I & active layer & flux & capacity \\
II & adapted active layer & flux & capacity \\
III & active layer & entrainment-deposition & non-capacity \\
\hline
\end{tabular}

case, it is not possible to model processes of adaptation to capacity load [6]. Moreover, the intrinsic variability that exists in the sediment transport rate and that provides a diffusive character is also neglected [7].

The combination of the flow, morphodynamic, and sediment transport equations form a system of partial differential equations. This needs to be mathematically classified as a wellposed to be representative of the physical processes under study. To be well-posed a unique solution must exist which does not infinitely diverge for infinitesimal perturbations in the problem data [8]. If a problem is ill-posed it loses its predictive capabilities since an arbitrarily small deviation in the initial or boundary conditions causes a large deviation in the solution in an arbitrarily small time. The numerical solution of an ill-posed problem is characterized by the appearance and growth of unphysical oscillations [9] and it is a symptom that the system of equations lacks certain regularizing physical processes. For instance, Kelvin-Helmholtz instabilities [10] appear in the two-phase problem when viscosity is neglected.

The model composed by the Saint-Venant equations [11] representing one-dimensional hydrostatic flow, the active layer model representing the mass balance of sediment per size fraction, and a local closure relation for the sediment transport rate has been used to predict mixed-size sediment processes in river morphodynamics for half a century. For instance, it has been used to study downstream fining in rivers [12] and bed surface armoring [13]. Yet, it presents a severe drawback: it can become ill-posed [14, 15].

Chavarrias et al. [16] studied the conditions in which the Saint-Venant-Hirano model becomes ill-posed. Here we study possible solutions to the problem of ill-posedness. In Section 2 we present the active layer model and two possible alternatives and in Section 3 we compare the different models to the results of a laboratory experiment conducted under conditions in which the active layer model is ill-posed.

\section{Morphodynamic Models}

In this section we first present the active layer model (Section 2.1). In Section 2.2 we introduce an adapted active layer model that retains the basics concepts and it is always wellposed. The adapted active layer model stems from a mathematical analysis and not from a study of the physical processes the active layer model lacks. On the contrary, a physicallybased solution to the problem of ill-posedess is presented in Section 2.3 where we relax the hypothesis that the sediment transport rate adapts instantaneously to changes in the flow.

\subsection{Model I: Active Layer Model}

In the active layer model [2] the bed is discretized into two layers. The uppermost layer (the active layer) interacts with the flow. This means that the sediment transport rate depends on the grain size distribution in the active layer, and the sediment that is deposited mixes with that present in the active layer. A key assumption is that the active layer is vertically homogeneous, i.e., it does not have vertical stratigraphy. Thus, for instance, when the active 
layer thickness is proportional to dune height [e.g. 17], the dune processes are averaged and the active layer model predicts the evolution on a time scale larger than the one responsible for vertical mixing due to dunes. The substrate sediment in the layer below the active layer only interacts (i.e., there is flux to or from the active layer) in case of net aggradation or degradation.

Mass conservation per size fraction is given by:

$$
\frac{\partial M_{a k}}{\partial t}+f_{k}^{I} \frac{\partial\left(\eta-L_{a}\right)}{\partial t}+\frac{\partial q_{b k}}{\partial x}=0,
$$

where $x[\mathrm{~m}]$ and $t[\mathrm{~s}]$ are the spatial and temporal coordinates, respectively, $M_{a k}=F_{a k} L_{a}$ $[\mathrm{m}]$ is the volume of sediment in the active layer per unit of bed area, $F_{a k}[-]$ is the volume fraction content of sediment in the active layer, $L_{a} \mathrm{~m}$ is the active layer thickness, $f_{k}^{I}[-]$ is the volume fraction content at the interface between the active layer and the substrate, $\eta[\mathrm{m}]$ is the bed elevation, and $q_{b k}\left[\mathrm{~m}^{2} / \mathrm{s}\right]$ is the sediment transport per unit width including pores. Subscript $k$ indicates the size fraction.

The sediment transport rate is assumed at capacity (i.e., to adapt instantly to changes in flow). The equation is said to be in flux form since changes in bed elevation and grain size distribution are steered by gradients in the flux of the sediment transport rate. Table 1 summarizes the characteristics of this model.

\subsection{Model II: Adapted Active Layer Model}

Chavarrias et al. [16] obtained an analytical expression to discern whether the active layer model is well-posed or ill-posed. This expression is obtained from the eigenvalues of the characteristic polynomial of the system matrix representing the model. If an eigenvalue has a complex component the model is ill-posed. We here adapt the active layer model introducing a parameter $\alpha[-]$ that modifies the time scale of the mixing processes. The modified active layer equation is:

$$
\alpha \frac{\partial M_{a k}}{\partial t}+f_{k}^{I} \frac{\partial\left(\eta-L_{a}\right)}{\partial t}+\frac{\partial q_{b k}}{\partial x}=0 .
$$

The parameter $\alpha$ has a direct influence on the eigenvalues of the system such that, if the active layer model is ill-posed, we can compute the value of $\alpha$ that makes all eigenvalues to be real. Thus, the model is always well-posed.

\subsection{Model III: Entrainment-Deposition Model}

To obtain a physically-based solution to the problem of ill-posedness in the active layer model we relax the assumption that the sediment transport rate responds instantaneously to the flow. Treating the sediment transport rate as an stochastic process, Furbish et al. [18] derive an advection-diffusion equation of the particle activity $\Gamma[\mathrm{m}]$ defined as the volume of particles in motion per unit of bed area. We extend this concept to mixed-size sediment obtaining an advection-diffusion for the particle activity per grain size:

$$
\frac{\partial \Gamma_{k}}{\partial t}+\frac{\partial v_{p k} \Gamma_{k}}{\partial x}-\kappa_{k} \frac{\partial^{2} \Gamma_{k}}{\partial x^{2}}=E_{k}-D_{k}
$$

where $v_{p k}[\mathrm{~m} / \mathrm{s}]$ is the particle velocity, $\kappa_{k}\left[\mathrm{~m}^{2} / \mathrm{s}\right]$ is the diffusivity, and $E_{k}[\mathrm{~m} / \mathrm{s}]$ and $D_{k}$ $[\mathrm{m} / \mathrm{s}]$ are the sediment entrainment and depositional rates, respectively. Equation 3 is solved in combination with an entrainment-deposition formulation of the active layer model:

$$
\frac{\partial M_{a k}}{\partial t}+f_{k}^{I} \frac{\partial\left(\eta-L_{a}\right)}{\partial t}=D_{k}-E_{k}
$$




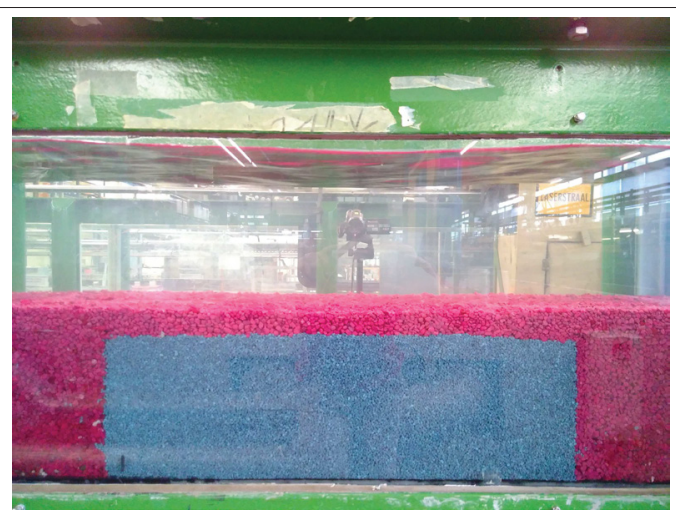

Figure 1. Initial condition of the experimental set-up. A patch of fine sediment (blue) is embed in coarse sediment (red).

The system is closed with relations for the entrainment and depositional rates [e.g. 19].

This generalization of the particle activity concept to mixed-size sediment does not take into consideration the interaction between particles of different grain size as regards to particle activity, velocity and diffusivity. The fact that, for instance, small particles move in average faster than coarse particles, gives rise to extra terms in Equation 3 that we neglect. This model can only be seen as a first attempt to gain insight into the physical processes that cause the active layer model to be ill-posed.

\section{Comparison of the Model Results with Laboratory Data}

In this section we compare the prediction of the three models (Section 3.2) to the results of a laboratory experiment (Section 3.1). We numerically solve the systems of equations of the three models using the research code Elv [20].

\subsection{Laboratory Experiment}

We have conducted a laboratory experiment under conditions in which the active layer model is ill-posed. We use a $14 \mathrm{~m}$ long, $0.40 \mathrm{~m}$ wide straight flume in the Water Laboratory of the Faculty of Civil Engineering and Geosciences of the Delft University of Technology. The sediment mixture is composed of two size fractions (fine and coarse) with characteristic sizes equal to $2.1 \mathrm{~mm}$ and $5.5 \mathrm{~mm}$, respectively. The bed is initially flat and composed of the coarse size fraction only but a $0.50 \mathrm{~m}$ patch of fine sediment at the center of the flume below a layer $4 \mathrm{~cm}$ thick of coarse sediment (Figure 1). Coarse sediment is fed at a constant rate and the water discharge remains constant during the experiment. The initial bed slope is in equilibrium with the water discharge and feed rate. A lowering of the downstream water level at a constant rate of $1 \mathrm{~cm} / \mathrm{h}$ over a period of $8 \mathrm{~h}$ causes degradation and, eventually, the entrainment of the fine sediment from the patch.

Sediment is transported in low bedforms ( 3 to 4 grain sizes in height). When the trough of a bedform exposes fine sediment a downstream propagating erosional wave forms. The coarse sediment load coming from upstream eventually covers the erosional pit (Figure 2ab). This implies that fine sediment is cyclically entrained. 


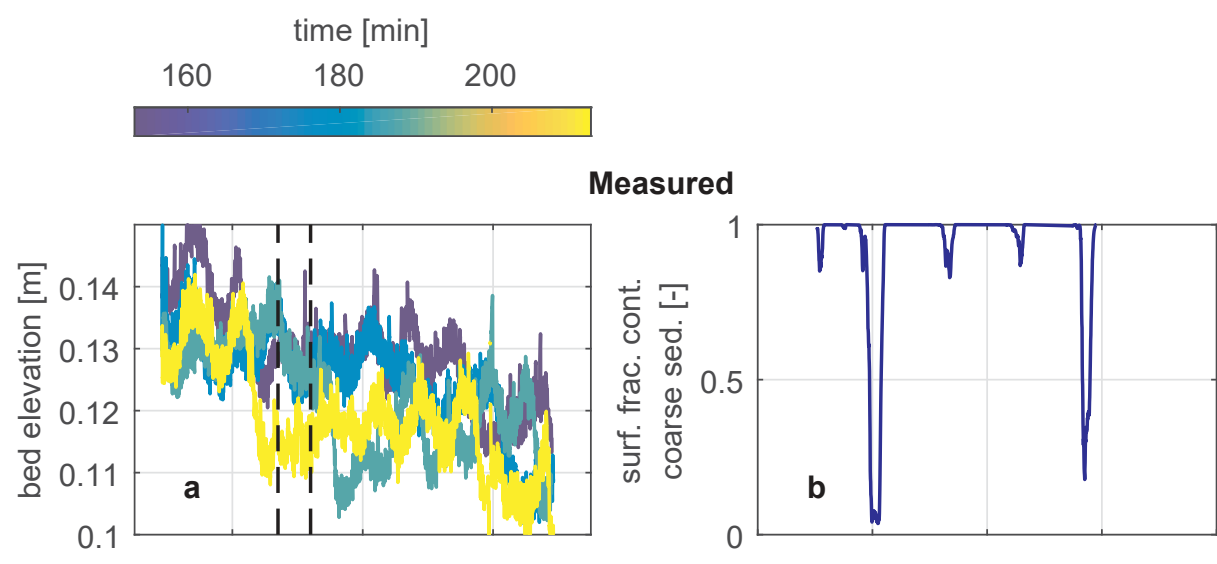

\section{Model I}
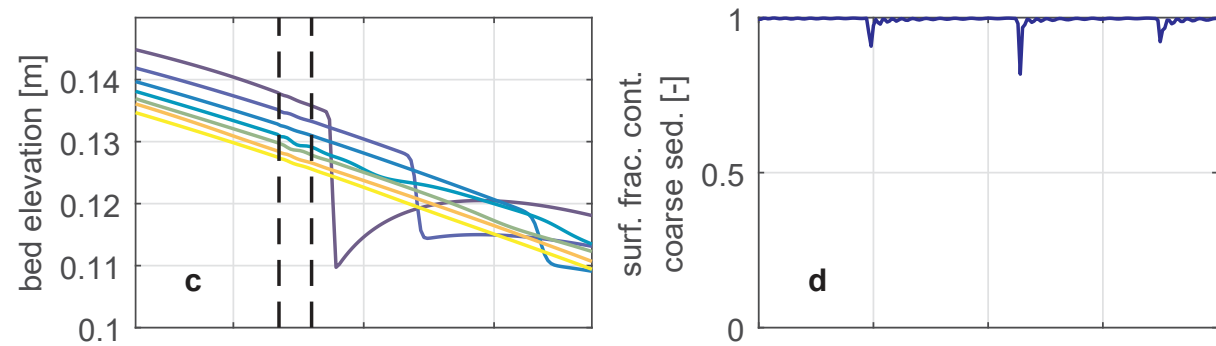

\section{Model II}
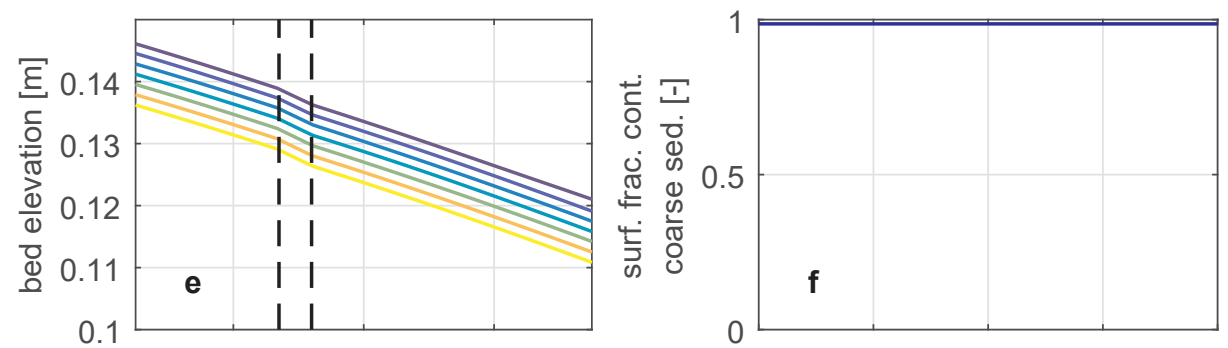

\section{Model III}
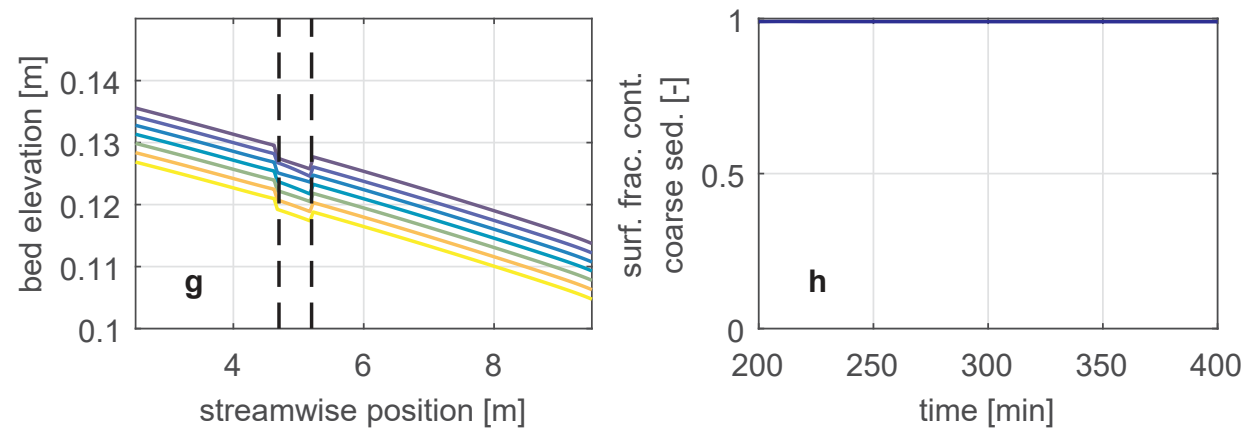

Figure 2. Bed elevation (left column) and surface volume fraction content of the coarse sediment at the patch (right column) measured (first row), predicted using the active layer model (second row), predicted using the regularized active layer model (third row), and predicted using the entrainmentdeposition model (fourth row). The dashed lines on the plots of the left column mark the position of the patch with fine sediment. 


\subsection{Results}

The active layer model (Model I) appears to be ill-posed when the fine sediment is at the interface between the active layer and the substrate. When this occurs, the numerical solution presents oscillations (Figure 2c-d). These oscillations are non-physical and their growth rate depends on the spatial discretization. The same simulation using a smaller cell size causes oscillations to grow faster [9].

The adapted active layer model (Model II) is well-posed and does not present oscillations (Figure 2e-f). The entrainment of fine sediment occurs in a continuous manner. The solution is representative of the average measured data, yet, it does not capture the small scale variability and the cyclic entrainment of fine sediment.

Model III is also well-posed and the solution is stable (Figure $2 \mathrm{~g}-\mathrm{h}$ ). Similarly to the adapted active layer model, it does not capture the small scale variability. The predicted volume fraction content at the bed surface is constant in time, like in Model II. The main difference between the models II and III occur in the predicted bed elevation along the patch. Model III predicts a bed level step not predicted by Model II. We cannot compare to the measured data since the predicted bed level step is of the order of $2 \mathrm{~mm}$ which is significantly smaller than the measured bed elevation variability (i.e., bed forms) and even smaller than the smallest grain size.

\section{Discussion and Conclusions}

The active layer model used to predict river morphodynamics with mixed-size sediment becomes ill-posed under certain circumstances. We have studied two possible solutions to this problem. The first solution is based on the introduction of a parameter that controls the celerity of the mixing processes. Use of a suitable value of this parameter guarantees that the active layer model is well-posed. The results of this model seem realistic when compared to measured data of a laboratory experiment conducted in conditions in which the active layer model is ill-posed. The adapted active layer model captures the time average results but it does not capture the measured small scale variability. Future work will compare the outcome of this model in reproducing other experiments.

The second solution is based on the relaxation of the assumption that the sediment transport rate adapts instantly to changes in flow. We generalize the particle activity concept developed from a stochastic view of sediment transport to mixed-size sediment conditions. The model is well-posed and the predictions are similar to the ones of the adapted active layer model. This second model is currently under development.

\section{References}

[1] F.M. Exner, Akad. Wiss. Wien Math. Naturwiss 129(2a), 929 (1920), (in German)

[2] M. Hirano, Trans. Jpn. Soc. Civ. Eng 195, 55 (1971)

[3] H. Sternberg, Zeitschrift für Bauwesen 25, 483 (1875), (in German)

[4] G. Parker, P.C. Klingeman, Water Resour. Res. 18, 1409 (1982)

[5] E. Meyer-Peter, R. Müller, Formulas for bed-load transport, in Proc. 2nd Meeting Int. Assoc. Hydraul. Struct. Res. (Stockholm, 1948), pp. 39-64

[6] R.G. Bell, A.J. Sutherland, J. Hydraul. Eng. 109 (1983)

[7] J.C. Roseberry, M.W. Schmeeckle, D.J. Furbish, J. Geophys. Res., Earth Surface 117, F3 (2012) 
[8] J.S. Hadamard, Lectures on Cauchy's problem in linear partial differential equations (Yale University Press, New Haven., 1923)

[9] D. Joseph, J. Saut, Theor. Comput. Fluid Mech. 1, 191 (1990)

[10] L.S.W.T.F.. Kelvin, Philos. Mag. 42, 362 (1871)

[11] A.J.C.B. Saint-Venant, Comptes Rendus des séances de l'Académie des Sciences 73, 237 (1871), (in French)

[12] A. Blom, E. Viparelli, V. Chavarrías, Geophys. Res. Lett. 43, 1 (2016)

[13] I. Park, S.C. Jain, J. Hydraul. Eng. 113, 845 (1987)

[14] J.S. Ribberink, Ph.D. thesis, Delft University of Technology, The Netherlands (1987)

[15] G. Stecca, A. Siviglia, A. Blom, Water Resour. Res. 50, 7563 (2014)

[16] V. Chavarrías, G. Stecca, R.J. Labeur, A. Blom, (submitted to Advances in Water Resources)

[17] R. Deigaard, J. Fredsøe, Nord. Hydrol. 9, 7 (1978)

[18] D.J. Furbish, P.K. Haff, J.C. Roseberry, M.W. Schmeeckle, J. Geophys. Res., Earth Surface 117, F03031 (2012)

[19] R. Fernandez-Luque, R. Van Beek, J. Hydraul. Res. 14, 127 (1976)

[20] A. Blom, V. Chavarrías, R.I. Ferguson, E. Viparelli, Geophys. Res. Lett. (2017) 